\title{
Sclerodermiform syndrome in oncological patient: The skin as a clinical herald case report
}

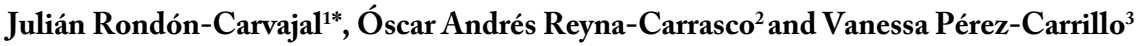 \\ ${ }^{1}$ Department of Internal Medicine, Ponficia Universidad Javeriana, Colombia \\ ${ }^{2}$ Department of Internal Medicine, Ponficia Universidad Javeriana, Colombia \\ ${ }^{3}$ Faculty of Medicine, Universidad Pontificia Bolivariana, Colombia
}

\begin{abstract}
Paraneoplastic skin syndromes include a wide spectrum of injuries that are clinically diverse and can present as vesiculoampullous diseases, erythrodermias, exanthema, keratoderma, among others. The cutaneous lesions can precede, be concomitant or follow a neoplastic disease; in fact, it is a rule within the oncological practice that after detecting a cutaneous lesion considered with high potential of malignancy, the appropriate complementary evaluations and exams are carried out to rule out this clinical scenario. We present the case of a patient with a solid tumor (prostate adenocarcinoma) in an advanced stage, in which after the diagnosis of the neoplasia, scleroderma lesions were documented as a probable paraneoplastic manifestation of late appearance, after ruling out previous exposure to alkylating agents, taxanes, platins or other chemotherapeutic agents such as bleomycin. Finally, a brief review of the literature on this association is made.
\end{abstract}

\section{Introduction}

The association between sclerodermiform syndromes and cancer has been described historically since the 1950s, in most cases preceding the debut of the neoplastic process $[1,2]$. The spectrum of the clinical manifestations includes from the classical picture of systemic sclerosis to cases of scleroderma and scleromyxedema. To date, less than 10 cases of this type of paraneoplastic skin syndromes related to prostate cancer have been reported $[3,4]$.

From the physiopathological point of view, it has been postulated that the increase in collagen deposition in the dermis is due to the high production of several cytokines by tumor cells, such as TGF-b (transforming growth factor beta) as well as to the formation of autoantibodies against RNA polymerase I-III [5]. Likewise, there have been reported cases of resolution of cutaneous manifestations once the tumoral progression is controlled [3,5]. Therefore, the objective of the case report is to sensitize the clinician about this unusual cutaneous paraneoplastic association (described until in 3-7\% of patients with solid tumors, mainly breast cancer) $[1,6]$ and to provide elements for an early and correct diagnostic orientation.

\section{Clinical case}

A 78-year-old man with a history of Gleason $5+3$ prostate cancer diagnosed in October 2015, in management with leuprolide acetate (7.5 mg IM (intramuscularly) each month), without other known pathological or pharmacological antecedents, who consulted the emergency department for an 8-month evolution, consisting of functional class deterioration, asthenia and adinamia in the context of hypochromic microcytic anemia and thrombocytopenia documented 2 weeks ago, with no known blood loss. Additionally, for 1 month he had been presenting limitations in oral opening and a sensation of "hardening of the skin on the face and hands that limits mobility".

In the revision by systems, it showed a loss of $10 \mathrm{~kg}$ of weight in approximately 6 months and ulcerated lesions in several limbs, with incomplete Raynaud's phenomenon, conserving only the phases of pallor and cyanosis of predominance in the right hand, without periungual telangiectasias. On physical examination, microstomy was described, as well as decrease in skin turgor, being more evident in the region of the nasolabial fold and forehead (Figures 1A and 1B). No peripheral adenopathy or visceromegaly was documented, nor were any findings relevant to the cardiovascular examination.

It was initially focused as a probable case of systemic sclerosis diffuse variety. The autoantibody profile was negative (anti-nuclear antibodies or ANAS, specific extractable nuclear antigen antibodies or ENAS), without deterioration in renal function or active sediment in uroanalysis, without complement consumption (C3, C4 fractions). Endoscopic studies were negative for active bleeding and bone scan reported disseminated osteoblastic disease in axial and appendicular skeleton, compatible with advanced metastatic involvement of prostatic adenocarcinoma, without evident pathological fractures.

In view of the bicytopenia evident from the admission, peripheral blood spread was performe, with presence of dacryocytes $(++)$ without other morphological alterations, being interpreted as myeloptisis in scenario of advanced invasive prostate cancer in presence of bicytopenia (anemia, thrombocytopenia). Oncological emergencies such as spinal cord compression syndrome, malignant hypercalcemia and even tumor lysis syndrome were ruled out.

${ }^{*}$ Correspondence to: Julián Rondón-Carvajal, Department of Internal Medicine, Ponficia Universidad Javeriana, Colombia, E-mail: julianrondoncarvajal@ gmail.com

Key words: cutaneous sclerosis, paraneoplastic syndromes, prostate cancer, dermatology

Received: October 06, 2020; Accepted: October 22, 2020; Published: October 27,2020 


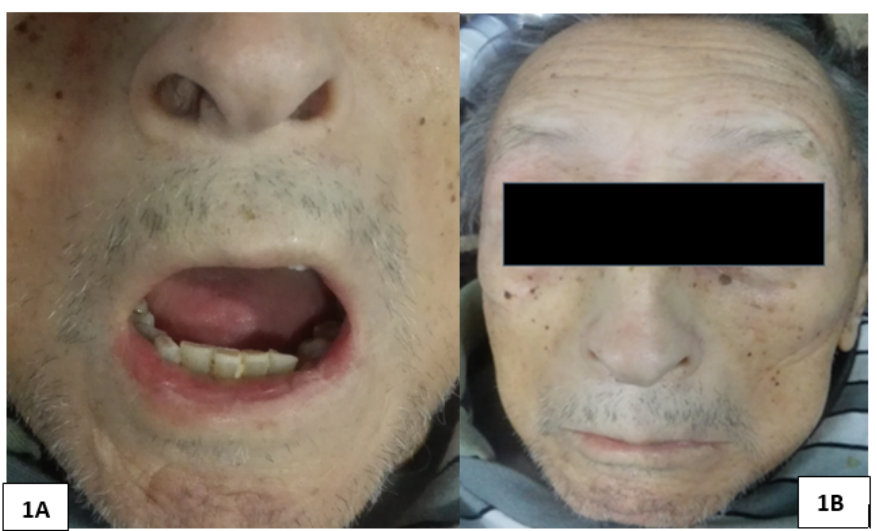

Figures 1A. Microstomy with limitation of the oral opening to $2.5 \mathrm{~cm}$. 1B. Reduction of the size of the nose, sharp in the tip or "Aquiline", inexpressive facies with prominent cheekbones, loss of nasogenian furrow and scarce wrinkles mainly in the forehead (statuary facie)

Complementary negative tomographic studies for distant metastatic lesions. Incidentally, a hypodense lesion dependent on the left thyroid lobe of approximately $26 \mathrm{~mm}$ was documented, which was later studied with fine needle aspiration biopsy (FNAB), being negative for malignancy.

The clinical evolution was stationary, with no indication for immunomodulatory therapy by rheumatology. Blood products were transfused and support management with multimodal analgesia and hydration guided by strict water balance was indicated. The patient discharged for palliative care management at home. The patient finally died out of hospital, 4 months after his discharge.

\section{Discussion}

Scleroderma syndromes are defined by the presence of cutaneous sclerosis, that is, an increase in the consistency of the elements that make up the dermis and/or hypodermis in relation to the presence of abundant collagen deposits in the dermis (cutaneous fibrosis), which leads to induration and loss of skin elasticity. Within the group of diffuse scleroderma syndromes, we include scleromyxedema, nephrogenic systemic fibrosis, Buschke's scleredema, POEMS syndrome, primary amyloidosis, chronic graft-versus-host reaction and those associated with toxins such as bleomycin and gemcitabine $[4,6]$.

Unlike systemic sclerosis, these syndromes are clinically different, being associated to low prevalence of multiorgan involvement, absence of symmetric Raynaud's phenomenon and low prevalence of specific antinuclear antibodies (anti ScL-70, anti RNP type). Likewise, there are sclerodermiform cutaneous hereditary presentations in adulthood (Werner's syndrome, Huriez scleroatrophy), whose early detection should also lead to the development of cancer in the medium and long term [6].

One of the largest patient series that sought to evaluate the association between scleroderma and cancer was published in 1979 by Duncan, et al. [7], which included 2141 patients in total assessed at Mayo Clinic between 1959 and 1975. Only 3 patients with prostate cancer, who later developed scleroderma lesions, were described in this series. The most frequently described neoplasms were lung and colon in men, and breast and uterus in women.

In a series of cases of rheumatological syndromes associated with cancer, Hakkou, et al. [8] presented the case of a 63 year old man who had been followed for 4 years for a prostate adenocarcinoma and who presented Raynaud's phenomenon in hands and feet, in addition to dyspnea grade 3 of the NYHA score, weight loss of $10 \mathrm{~kg}$ in 1 year and subsequent skin sclerosis, acropaquia and edema in a distribution of gloves and socks. In this case, antinuclear antibodies were positive (1/340) with spotted fluorescence and anti-SCL-70 antibodies. The patient finally died, following a course very similar to our clinical case.

Most of the series of cases described suggest that the appearance of sclerosing lesions in patients older than 60 years should be oriented towards underlying active neoplasia, taking into account the evidence to date that relates the presence of anti-RNA polymerase III autoantibodies with the future appearance of cancer [9], due to somatic mutations in the gene that encodes for this protein (POLR3A), which in turn induces an aberrant $\mathrm{T}$-cell mediated response against the tumor.

Finally, Kikuchi, et al. [10] reported 71 cases of patients with scleroderma lesions, 5 (7\%) of which were associated to neoplasia. None of them had Raynaud's phenomenon, esophageal involvement or antinuclear antibodies against ScL-70 (topoisomerase I). Although initially it may become indistinguishable from systemic sclerosis, scoliodermiform syndrome or pseudosclerosis tends to have a more rapid progression, with variable inflammatory infiltration in the dermis. Likewise, in these cases it was possible to measure serum levels of FGFb (basic fibroblast growth factor) by ELISA, being higher in patients with sclerosing lesions associated with neoplasms than in patients with systemic sclerosis in its limited and diffuse varieties.

\section{Conclusion}

- The concurrence between cancer and sclerosing lesions can have 4 possible scenarios: that they occur independently, that sclerosis precedes the appearance of neoplasia, that neoplasia induces the appearance of sclerosis and that immunosuppressive therapy for the management of systemic sclerosis leads to the development of the tumor, as in the case of some cytotoxic agents.

- Although polymyositis/dermatomyositis represents the most frequently cited rheumatological entity in the spectrum of paraneoplastic rheumatological manifestations, it is important for the clinician to recognize the different entities grouped in the group of scleroderma syndromes, since this will allow them, in certain scenarios such as in adults over 60 years old, to anticipate the presentation of neoplasic processes, with the prognostic implications that this entails.

- Given its atypical behavior in clinical terms and in laboratory findings, this case report is shared in order to extend the experience of the different cutaneous paraneoplastic syndromes associated to solid tumors, in different moments of the natural history of oncological disease.

\section{Authorship}

All listed authors have made substantive intellectual contributions to the case follow-up, manuscript design and bibliographic research.

\section{Acknowledgments}

None.

\section{Financial support}

None. 


\section{Ethical aspects}

The patient offered an informed consent to this publication and anonymity was assured. The authors have an original document with the patient's signature, authorizing the publication of the clinical case.

\section{Conflicts of interests}

The authors declare no conflicts of interests.

\section{References}

1. Rovisco J, Serra S, Abreu P, Coutinho M, Santiago T, et al. (2014) Paraneoplastic sclerodermiform syndrome--case report. Acta Reumatol Port 39: 87-90.

2. Marek M, Rudny R (2016) Scleroderma of geriatric age and scleroderma-like paraneoplastic syndrome- description of two cases. Reumatologia 54: 91-94.

3. Jablonska S, Blaszczyk M (1998) Scleroderma-like disorders. Semin Cutan Med Surg 17: $65-76$.
4. Marasini B, Conciato L, Belloli L, Massarotti M (2009) Systemic sclerosis and cancer. Int J Immunopathol Pharmacol 22: 573-578.

5. Shah AA, Casciola-Rosen L (2015) Cancer and scleroderma: a paraneoplastic disease with implications for malignancy screening. Curr Opin Rheumatol 27: 563-570.

6. Ciołkiewicz M, Domysławska I, Ciołkiewicz A, Klimiuk PA, Kuryliszyn-Moskal A (2008) Coexistence of systemic sclerosis, scleroderma-like syndromes and neoplastic diseases. Pol Arch Med Wewn 118: 119-126.

7. Duncan SC, Winkelmann RK (1979) Cancer and scleroderma. Arch Dermatol 115: 950-955.

8. Hakkou J, Rostom S, Bahiri R, Hajjaj-Hassouni N (2012) Paraneoplastic rheumatic syndromes: report of eight cases and review of literature. Rheumatol Int 32: 1485-1489.

9. Moinzadeh P, Fonseca C, Hellmich M, Shah AA, Chighizola C, et al. (2014) Association of anti-RNA polymerase III autoantibodies and cancer in scleroderma. Arthritis research \& therapy $16: \mathrm{R} 53$.

10. Kikuchi K, Hoashi T, Yazawa N, Tamaki K (2006) Pseudoscleroderma associated with cancer. Clin Exp Dermatol 31: 381-383.

Copyright: (C2020 Rondón-Carvajal J. This is an open-access article distributed under the terms of the Creative Commons Attribution License, which permits unrestricted use, distribution, and reproduction in any medium, provided the original author and source are credited. 